\title{
Breve historia de la cirugía en Yucatán.
}

\author{
Historia de la Medicina
}

Alejandro Cervera-Andrade*.

Escuela de Medicina, Universidad de Yucatán, Mérida, Yucatán, México.

\section{RESUMEN.}

Por las fuentes históricas más antiguas sabemos que los mayas conocían y usaban muchas hierbas para curar sus enfermedades, para cicatrizar sus heridas y para curar las mordeduras de víboras. Algunas de estas prácticas perduran hasta hoy. Los primeros médicos que vinieron a Yucatán fueron Maese Iñigo López, cirujano, que vino con la primera expedición de Montejo; Juan del Rey, herbolario, médico y cirujano, quien previamente había estado en Guatemala. Durante la Colonia figuraron: Fray Gaspar de Molina, Fray Gabriel de San Buenaventura; Fray Andrés de Avendaño. En el siglo XVIII figuraron cuatro médicos ilustres: Juan Pereyra, portugués, Juan Francisco Mayoli, italiano romano que ejerció en Valladolid y a quien se atribuye la paternidad del Libro del Judío, Lucas Teniere, (francés) y Claudio Grandel, (francés), en Campeche, en 1720. A fines del siglo XVIII figuraron: Fray Antonio de Vecaría, franciscano y Francisco León de Galera, en Mérida, y en Campeche Carlos Escoffié y Grevy, francés, y Benjamín Bothe, inglés. A comienzos del siglo XIX

* Publicación póstuma. Trabajo presentado en la Academia Nacional de Cirugía, en la sesión del día 25 de Agosto de 1942. vino de Europa el doctor Alejo Dancourt en el año 1802; en 1805 llegó a Campeche el doctor Juan Antonio Frutos, español, y por la misma época figuraron en dicha ciudad el Dr. Claro José Beraza y Monsieur Renón, médico francés. En la época de la Independencia, destacaron don Eusebio Villamil, sacerdote católico, don Sebastián Sotomayor, español; don Manuel S. Howard, norteamericano; don Joaquín Muñoz y Ramírez, queretano; don José Matilde Sansores, y una comadrona que tuvo mucha fama, doña Nicolasa Treviño.

En 1846, llegó a Mérida don José María Tappam, de la Universidad de Harvard, y fue profesor de la Escuela de Medicina durante muchos años; se le atribuye el mérito de haber practicado varias amputaciones, por primera vez en Yucatán, así como la talla pireneal para la extracción de cálculos de la vejiga. En la misma época el Dr. S. Cabot, practicó por primera vez la operación de la catarata. En el año de 1847, 4 de junio, en el Hospital de San Juan de Dios de la ciudad de Mérida, el Dr. José Matilde Sansores dio la primera anestesia general con éter. En el año de 1852, el Dr. Manuel Campos González hizo por primera vez en Campeche la operación de catarata. El cirujano

Solicitud de sobretiros: Revista Biomédica, Av. Itzáes No. 490 x 59, C.P. 97000, Mérida, Yucatán, México. Fax. (999) $923-61$-20 Aceptado para publicación 12/Marzo/2002 


\section{A Cervera-Andrade.}

yucateco don José Ricardo Sauri, llegó a Mérida en 1870 y operó por primera vez el pterigion, la iridectomía por glaucoma, la enucleación del globo ocular y operó gran número de cataratas. El Dr. Manuel Arias Durán hizo la primera sinfisiotomía y el Dr. Augusto Molina Solís la primera operación de hernia estrangulada, en el año de 1885.

En 1884 el Dr. Tappam operó por primera vez un absceso de mama. En 1887 un cirujano extranjero hizo la primera extirpación de un quiste del ovario, el Dr. Sauri practicó una operación de apendicitis, de mastoiditis y en 1894 la primera talla hipogástrica. En 1895 el Dr. Saturnino Guzmán Cervera operó el primer caso de herida penetrante de abdomen y en 1897 el Dr. Teodosio Pérez Peniche hizo la primera anestesia raquídea. A fines del siglo XIX el Dr. Ramón Albert Pacheco operó el primer caso de un embarazo extrauterino, el Dr. Eudaldo Ferráez hizo la primera operación cesárea y el Dr. Guzmán el primer raspado uterino postabortum. En 1901 el Dr. José Ricardo Sauri hizo la primera histerectomía. Durante los primeros cuatro decenios del siglo XX diferentes intervenciones quirúrgicas se realizaron por vez primera en Yucatán. Entre ellas destacan: gastroenterostomía por vía transperitoneal, abocamiento abormal del recto con perforación del ano, citoscopía y cateterismo uretal, injerto glandular por el método de Voronof, operación de la Fort, histerectomía por vía perineal, nefrectomía por vía transperitoneal, extirpación de la glándula tiroides, ventrofijación del muñón resultante de histerectomía subtotal, operación de Calot y operación de Steinach.

(Rev Biomed 2002; 13:144-151)

Palabras clave: Historia de la Medicina, cirugía, Yucatán.

\section{SUMMARY.}

\section{A brief history of surgery in Yucatan.}

From the oldest historical sources we know that Mayans knew of and used many herbs to cure diseases, to close wounds and to cure snake bites. Some of these practices continue to this day. The first doctors to come to Yucatan were Maese Iñigo López, surgeon, who arrived with Montejo's first expedition; Juan del Rey, herbolist, doctor and surgeon who had previously been in Guatemala. During the colonisation, the most notable figures were Fray Gaspar de Molina, Fray Gabriel de San Buenaventura and Fray Andrés de Avendaño. In the 18th century there were four distinguished doctors, Juan Pereyra from Portugal, Juan Francisco Mayoli, an Italian who practiced in Valladolid and to whom the Book of the Jew is attributed, Lucas Teniere (French) and Claudio Grandel (French), in Campeche in 1720. At the end of the eighteenth century Fray Antonio de Vecaría, a franciscan monk and Francisco León de Gatera came to Mérida and Carlos Escoffié and Grevy (French) and Benjamen Bothe (English) came to Campeche. At the beginning og the 19th century Dr. Alejo Dancourt came from Europe in 1802, doctor Juan Antonio Frutos (Spanish), Dr. Claro José Beraza (Spanish) and Monsiour Renon, a French doctor arrivied in Campeche. During the era of the independence, Don Eusebio Vellamil a catolic priest, Don Sebastian Sotomayor, spaniard, Don Manuel S. Howard, american, Don Joaquín Muñoz y Ramírez from Queretaro, Don José Matilde Sansores and a midwife who became very famous, Doña Nicolasa Treviño, were all the doctors who stoud out.

In 1846, Don José María Tappam from the University of Merida arrived in Merida and was professor of the School of Medicine during many years, he has been given vredit for having carried out several amputations for the first time in Yucatan, as well as the removal of gall stones from the bladder. In the same era, Dr. S. Cabot practiced a cataract operation for the first time. On June 4, 1847 Dr. José Matilde Sansores applied the first general anaesthetic using ether at the hospital San Juan de Dios, Merida in 1852, Dr. Manuel Campos González practiced the first cataract operation in Campeche. The Yucatecan surgeon Don José

\section{Revista Biomédica}




\section{Historia de la cirugía en Yucatán.}

Ricardo Sauri arrived in Merida in 1870 and operated on the pterigion for glaucoma, enucleation of an eye and he operated on numerous cataracts. Dr. Manuel Arias Durán carried out the first sinfisiotomia and Dr. Augusto Molina Solís the first operation on a Strangulated hernia in 1885.

In 1884 Dr. Tappam operated on a breast absess for the first time. In 1887 a foreign surgeon removed an ovarian cyst, Dr. Sauri practiced on appendicectomy, mastoiditis and in 1894 the first hypogastric lichotomy. In 1895 Dr. Saturnino Gusmán Cervera operated on the first case of a deep wound in the abdomen and in 1897, Dr. Teodosio Pérez Peniche performed the first rachialgia. At the end of the nineteenth century Dr. Ramón Albert Pacheco operated on the first case of an extrauterine pregnancy, Dr. Eudaldo Ferráez performed the first cesaraen and Dr. Guzmán did the first post abortion scrape. In 1901 Dr. José Ricardo Sauri carried out the first hysterectomy. During the first four decades of the twentieth century, different surgical interventions were performed for the first time in Yucatan. Among them, the following stand out the most; transperitoneal gastroenterostomy, abnormal rectum abocamiento with anal perforation, uretal cystoscopy and cateterism, glandular graft using Voronof's method, fort operation, perineal hysterectomy, transperitoneal nefrectomy, removal of the thyroid gland, ventrofixation of the stump in a partial hyst hystomrectomy Calot's operation and Steinach's operation.

(Rev Biomed 2002; 13:144-151)

Key words: History of Medicine, surgery, Yucatan.

\section{INTRODUCCIÓN.}

La Península de Yucatán, actualmente dividida en tres entidades: Yucatán, Campeche y Quintana Roo, fue asiento del gran Imperio Maya, antes de la Conquista, y constituyó una Capitanía General durante la Dominación Española. Así pues, al abordar cualquier tema histórico relacionado con
Yucatán -historia antigua- forzosamente necesitaremos referirnos también a Campeche, cuya capital, la ciudad del mismo nombre, tuvo gran importancia, como la tuvo también Valladolid. Ambos estados hermanos geográficamente, lo están también por razones históricas y etnológicas.

Las fuentes históricas más antiguas que nos pueden proporcionar datos acerca del tema que tratamos de desarrollar, son las "Relaciones" enviadas a España por los conquistadores -tanto soldados como los religiosos que con ellos vinieron- documentos recopilados en archivos y después publicados por los historiadores vernáculos. Por ellos sabemos que los mayas conocían y usaban muchas hierbas para curar sus enfermedades, para cicatrizar sus heridas y para curar las mordeduras de víboras; utilizaban el "padz" o masaje para componer los huesos luxados, para sus casos obstétricos y aún más para ciertos cólicos atribuidos a "torsión del cirro" (ignoramos a qué órgano aplicaban esta denominación); utilizaban el "pedz" -apesgar o apretar- en ciertas afecciones, principalmente en las hinchazones, bien directamente aplicando los dedos o la palma de la mano, o interponiendo alguna superficie plana y resistente como las hojas del "chunup", utilizaban también el "jub" -punzadora con púas de puercoespín- para curar algunos dolores neurálgicos, utilizaban el "coh-cán", sangría que practicaban introduciendo debajo de la piel de la frente colmillos secos de "cascabel" (serpiente), sirviendo el canal del colmillo a manera de cánula o trócar. Utilizaban los baños fríos para bajar las fiebres y baños calientes de vapores de hierbas salcochadas para muchas dolencias. Las personas que ejercían este arte eran consideradas como médicos-hechiceros o médicos sacerdotes y recibían diversos nombres: "dzac-yah", "h-men", "kax-bac", y sus curaciones iban siempre acompañadas de ciertas ceremonias, posiblemente para ejercer mayor influencia en la mente del enfermo. Algunas de estas prácticas perduran hasta hoy entre nuestros indios; con o sin intervención del hmen, las heridas son curadas con apósitos de 


\section{A Cervera-Andrade.}

hierbas machacadas o bien con polvos de hierbas carbonizadas después de lavarlas con el cocimiento de algunas hojas o raíces.

\section{LOS MÉDICOS DE LA ÉPOCA COLONIAL.}

De la actuación de los primeros médicos españoles que vinieron a la península, solamente podemos citar nombres: Maese Iñigo López, cirujano, vino a estas costas peninsulares con la primera expedición de Montejo y murió pocos días después de desembarcar; Juan del Rey, herbolario, médico y cirujano, quien después de haber estado en Guatemala vino con Montejo a Champotón, le acompañó en la conquista de Yucatán y luego se estableció en Mérida en el año de 1542. Durante los tres siglos de Dominación Española figuraron: Fray Gaspar de Molina, franciscano, ejercía la profesión no sólo en el Monasterio donde había instalado su botica sino también entre los habitantes de la ciudad; siglo XVII: Fray Gabriel de San Buenaventura; Fray Andrés de Avendaño, 1695.

En el siglo XVIII figuraron cuatro médicos ilustres: Juan Pereyra, portugués, que ejerció en Mérida; Juan Francisco Mayoli, italiano romano que ejerció en Valladolid y a quien se atribuye la paternidad del Libro del Judío, que trata del uso de hierbas yucatecas para curar las enfermedades (año 1713); Lucas Teniere, (francés) y Claudio Grandel, (francés), en Campeche, año 1720. De éstos el que tuvo más fama fue el italiano Mayoli, quien murió en Valladolid en 1770. A fines del siglo XVIII figuraron Fray Antonio de Vecaría, franciscano y Francisco León de Galera, en Mérida, y en Campeche Carlos Escoffié y Grevy, francés, y Benjamín Bothe, inglés.

A comienzos del siglo XIX, gobernando el Capitán General don Benito Pérez Valdelomar, hizo venir de Europa al doctor Alejo Dancourt en el año 1802; entre otros cargos desempeñó el doctor Dancourt el de Rector de la Universidad de Yucatán en la época en que se fundó la enseñanza de la Medicina en esta entidad; en 1805 llegó a Campeche el doctor Juan Antonio Frutos, español, y por la misma época figuraron en dicha ciudad el
Dr. Claro José Beraza y Monsieur Renón, médico francés.

\section{ÉPOCA INDEPENDIENTE.}

En la época de la Independencia, además del Dr. Dancourt, figuraron don Eusebio Villamil, sacerdote católico que recibió enseñanza médica del Dr. Dancourt; don Sebastián Sotomayor, español; don Manuel S. Howard, norteamericano; don Joaquín Muñoz y Ramírez, queretano; don José Matilde Sansores, y una comadrona que tuvo mucha fama, doña Nicolasa Treviño. En 1833 llegó a la Península el Dr. don Ignacio Vado Lugo, de Guatemala, con título de esa ciudad y de París.

El gobierno del Estado decretó la fundación de la Cátedra de Medicina en la Universidad y el Dr. Vado fue nombrado para desempeñarla. Un año después, en 1834, el Dr. Juan Hubbe, de la Universidad de Tubingen, recién llegado, es nombrado catedrático de vísperas de Cirugía y en el año 1835 don Manuel Campos, discípulo en Campeche de don Juan Antonio Frutos, recibió en la Universidad de Yucatán el título de Licenciado en Medicina, después de presentar exámenes en dicha Universidad. El Dr. Campos regresó a Campeche, se hizo cargo del Hospital y en unión del Dr. Domingo Duret fundó en dicha ciudad la Escuela de Medicina en 1849 con autorización del gobierno de Yucatán. Hasta aquí los datos históricos recogidos no hacen mención de haberse practicado más actos quirúrgicos que curaciones de heridas y atención de partos, y si alguna amputación se practicó no lo sabemos con certeza, sino solamente por el relato de un anciano de Valladolid que da la noticia de haber oído referir el hecho de haberse practicado una amputación de la pierna a un carretero, por un señor llamado Juan Leston en Valladolid, a mediados del siglo XVIII, y que dicho señor se hacía llamar con el título de "físico".

En 1846, llegó a Mérida un médico y cirujano norteamericano, don José María Tappam, de la Universidad de Harvard, se incorporó a la Universidad Yucateca y fue profesor de la Escuela

\section{Revista Biomédica}




\section{Historia de la cirugía en Yucatán.}

de Medicina durante muchos años; se distinguió como partero y se le atribuye el mérito de haber practicado varias amputaciones, posiblemente por primera vez en Yucatán, así como la talla pireneal para la extracción de cálculos de la vejiga, habiendo ejercido la profesión durante 60 años. Por esa época vino a Yucatán acompañando a Mr. Stephens un cirujano norteamericano, el Dr. S. Cabot, de quién se refiere que practicó por primera vez la operación de la catarata. En el año de 1847, 4 de junio, en el Hospital de San Juan de Dios de la ciudad de Mérida, el Dr. José Matilde Sansores dio la primera anestesia general con éter, un año después que Warren y Morton la hubieron aplicado en Estados Unidos, el enfermo se llamaba José María Huchín y la operación consistió en la amputación de la mano izquierda, destruida por la explosión de una bomba, siendo operadores el Dr. Tappam y el Dr. Sansores. En el año de 1852, el Dr. Manuel Campos González hizo por primera vez en Campeche la operación de catarata en la persona del Sr. Pedro Segovia. Y en el mismo año hizo por primera vez la talla perineal en Mérida el Dr. Tappam.

En 1869, el inolvidable médico y cirujano yucateco don José Ricardo Sauri, llegó a Mérida después de graduarse en el College Bellevue y en la Facultad de París y en 1870 ingresó como catedrático y jefe del Servicio de Cirugía, desempeñando el cargo durante 15 años consecutivos habiéndose distinguido en esa época como cirujano especializado en cirugía ocular. Fue quien operó por primera vez el pterigion, la iridectomía por glaucoma, la enucleación del globo ocular y operó gran número de cataratas, lo que conquistó gran fama. En esa época, el Dr. Manuel Arias Durán hizo la primera sinfisiotomía y el Dr. Augusto Molina Solís la primera operación de hernia estrangulada, en el año de 1885, en la persona del padre Bello, cura del Sagrario.

De 1886 a 1888, Molina y Sauri, cirujanos del hospital de Mérida Yucatán, se dieron cuenta de lo absurdo que era tener en las mismas salas, enfermos de medicina y enfermos de cirugía, y dispusieron la separación de estas dos categorías de enfermos, tanto en hombres como en mujeres, creándose así, el Departamento de Cirugía con sus respectivas salas.

En el año de 1887, un nuevo elemento de indiscutible valor científico vino a sumarse a los existentes: El Dr. Saturnino Guzmán Cervera, yucateco-, quien después de graduarse en la facultad de Medicina de Mérida, hizo de nuevo estudios en la Facultad de París hasta obtener el título de dicha Facultad y pasando después a Viena y a Berlín para asistir a las clínicas más famosas de esas ciudades; fue alumno de los más renombrados cirujanos franceses y alemanes, de esa época en que Lister aplicando las teorías de Pasteur hizo el milagro de llevar la cirugía hasta alturas no sospechadas hasta entonces. Así es que al llegar a Mérida con tan vasto caudal de conocimientos, sus primeras operaciones fueron calificadas como atrevidísimas; llegó a Mérida en 1887 y en 1889 fue nombrado profesor de Clínica Médica implantando la enseñanza de la propedeútica, interrogatorio, palpación, auscultación, etc.; (antes del Maestro Guzmán, en nuestro medio se diagnosticaba la pulmonía por el esputo herrumbroso, pues no se conocía la auscultación). En 1891 obtuvo por oposición la cátedra de Clínica Quirúrgica y el servicio correspondiente, cargos que desempeñó durante cuarenta años consecutivos, pudiendo decirse que llenó una época e hizo escuela, pues con muy contadas excepciones todos los cirujanos jóvenes que figuran actualmente fueron discípulos suyos. Poco antes de ingresar el Dr. Guzmán al Hospital, el Dr. José Ricardo Sauri se separó de la cátedra y del Servicio por motivos políticos, entrando a sustituirlo el Dr. D. José Patrón Correa quien duró en dicho Cargo dos o tres años. Al separarse del Hospital el Dr. Sauri continuó su labor quirúrgica entre la clientela civil, dio frecuentes viajes a París, trajo la primera jeringa hipodérmica y estableció el primer sanatorio quirúrgico, al que denominó modestamente Casa de Salud, donde operaba especialmente casos ginecológicos; en su consultorio funcionó el primer 


\section{A Cervera-Andrade.}

aparato de rayos $\mathrm{X}$, traido a Yucatán por el Dr. Teodosio S. Pérez Peniche.

Prosigamos nuestra relación cronológica. En 1884 (aproximadamente) el Dr. Tappam operó por primera vez un absceso del seno (mama); en 1887 un cirujano extranjero (cuyo nombre no se conservó) hizo la primera extirpación de un quiste (enorme) del ovario con resultado fatal y poco después el Dr. Sauri practicó una operación de apendicitis en la persona de un niño de 10 años, con éxito (fue un caso de apendicitis supurada); en el mismo año operó (por primera vez) de mastoiditis a un Sr. Manzanilla; en 1894 hizo la primera talla hipogástrica: en 1895 el Dr. Guzmán operó el primer caso de herida penetrante de abdomen, por arma de fuego, en la persona de un turco (o sirio libanés) que después de asesinar a su mujer se disparó con intención de suicidarse, fue llevado inmediatamente al Hospital y una hora después del suceso fue hecha la laparotomía, habiéndole suturado doce perforaciones del intestino delgado, con éxito (caso citado en la tesis del Dr. Berrón); en 1897 el Dr. Teodosio Pérez Peniche hizo la primera anestesia raquídea con solución de cocaína. A fines del siglo XIX (sin poder precisar el año) el Dr. Ramón Albert Pacheco, entonces jefe del departamento) de mujeres, operó el primer caso de embarazo extrauterino. Siempre a fines del siglo XIX, el Dr. Eudaldo Ferráez hizo la primera operación cesárea y el Dr. Guzmán el primer raspado uterino postabortum, con buen resultado. En 1901 el Dr. Sauri hizo la primera histerectomía por fibroma, empleando procedimiento de Doyenm con éxito.

\section{LA ENSEÑANZA DE LA CIRUGÍA.}

Por lo que respecta a la enseñanza de la Cirugía y al progreso de la misma en el Hospital, copio lo que dice el Dr. Eduardo Aldana Barrera, en su libro "La Cirugía en Yucatán", acerca del particular: "En 1895, cuando finalizábamos nuestros estudios, las operaciones se practicaban en una pieza del piso bajo del ex-convento de la Mejorada (local que ocupaba el Hospital O'Horán) y a la cual se llamaba sala de operaciones; ésta era de cuatro metros de largo por tres y medio de ancho y tres y medio de alto; recibía luz por una puerta de dos y medio de alto que miraba al sur, y comunicaba en su extremo norte por otra puerta con una pieza un poco mayor llamada arsenal; del lado poniente estaba limitada por un muro sin puertas y del lado oriente con otra puerta que daba acceso a un angosto corredor por la que entraban el cirujano y los estudiantes. El arsenal era una especie de museo de instrumentos de cirugía que hablaban de las distintas épocas de la cirugía en la localidad; la mesa de operaciones era de madera; los instrumentos se pasaban por agua hirviendo y luego se sumergían en solución fenicada y más tarde en solución de bicloruro o de oxicianuro de mercurio; se usaban torundas humedecidas en estas soluciones para esponjar el campo operatorio; el cirujano se ponía una blusa no esterilizada, y así se practicaron con éxito muchísimas operaciones de cirugía general y algunas en el abdomen, como hernias y heridas penetrantes, tallas y trepanaciones y síntesis óseas"

De las memorias del Dr. Guzmán, publicadas en la revista local "Humanidad", el año pasado, tomo los siguientes datos: "En 1891, se hizo cargo el Dr. Guzmán de la cátedra y del Servicio de Cirugía; siguiendo lo que había visto en Europa, inauguró, adaptándose al medio, las lecciones de clínica quirúrgica tres veces por semana, con el enfermo a la vista, comenzando las lecciones con la propedeútica quirúrgica; sustituyó el ungüento amarillo y las hilas por las soluciones fenicada o de mercurio, y torundas empadadas de estas soluciones y después compresas de gasa; para hacer las esterilizaciones trajo de París una estufa seca de Pean (o de Pupinel); enseñó a los alumnos la canalización de las cavidades supurantes con tubos de gaucho y oclusión completa, la curación radical de las hernias en general, la uretrotomía interna como acto preliminar para las dilataciones de la uretra con las bujías Beniqué, la uretrotomía externa y el cateterismo retrógado en los casos de ruptura traumática de la uretra; la talla hipogástrica,

\section{Revista Biomédica}




\section{Historia de la cirugía en Yucatán.}

haciéndoles ver las ventajas de ésta sobre la talla perineal, la extirpación de la próstata por el método de Freyer; la operación de empiema, la toracoplastía, la operación del absceso hepático, la nefrostomía, la nefrectomía, la reducción de las luxaciones por medio de maniobras fundadas en la anatomía de la región, la sutura metálica de la rótula fracturada, la trepanación longitudinal en la osteomielitis, la operación de la mastoiditis, la trepanación del cráneo en determinados casos de fractura, la apendicectomía, la traqueotomía, el raspado uterino post abortum, los injertos dermoepidérmicos en las grandes superficies en supuración, el método de Ollier de injertos cutáneos, la cincunducción de las úlceras callosas, la simpatectomía femoral, la resección de las úlceras varicosas y su tratamiento por medio de las inyecciones esclerosantes". Por lo ennumerado anteriormente se puede juzgar la obra del maestro.

Hasta el año de 1902, el Dr. Guzmán era jefe del único servicio de cirugía; a partir de ese año, el Dr. D. Augusto Molina, director del Hospital, dividió el servicio de cirugía creándose el de mujeres y nombrándose al Dr. Manuel Palma Mena cirujano de dicho departamento.

El 6 de febrero de 1906 durante la visita presidencial del Gral. Díaz, siendo gobernador el Lic. Olegario Molina y director del Hospital el Dr. Augusto Molina, se inauguró el nuevo edificio del Hospital O'Horán, en el barrio de Santa Catalina, al poniente de la ciudad, hospital moderno, con 23 pabellones para enfermos, dos salas de operaciones con sus arsenales y salas para anestesia, un edificio para laboratorio de anatomía patológica, bacteriología y química médica, y otros para la administración y el cuerpo auxiliar facultativo, practicantes, arsenales, etc., es nombrado cirujano de mujeres el Dr. Ignacio Molina y se crea el segundo servicio de cirugía de hombres, para el que es designado el Dr. Eudaldo Ferráez, graduado en la Facultad de París. El Dr. Augusto Molina, con tesonero empeño organizó el nuevo establecimiento haciendo realidad la asepsia y la antisepsia; se trajeron autoclaves para esterilizar las compresas de gasa, las servilletas y campos operatorios y las blusas de los cirujanos; se adquirió un instrumental quirúrgico moderno, destinándose los antiguos de mango de madera al anfiteatro para las lecciones de medicina operatoria. Por sugerencia del Dr. Albert, se hizo venir a un bacteriólogo notable, el Dr. Haral Seidelin, médico danés, de la Escuela de Medicina Tropical de Liverpool, para enseñar a los alumnos, prácticamente la Bacteriología y Parasitología, y la Anatomía Patológica. Al dividirse el servicio de cirugía se hizo más fructífera la labor quirúrgica despertando legítimas y bienhechoras emulaciones en los profesores de las cátedras; con la fundación de los laboratorios, la labor de los cirujanos tenía la fiscalización científica y ya no el veredicto de la maledicencia; y como dice el Dr. Aldana: "la enseñanza se hizo efectiva; la teoría se aprendía verificándola".

En esa época, por iniciativa del Dr. Ferráez se hizo obligatorio para los estudiantes y practicantes el uso de las blusas clínicas y se comenzó a llevar las historias clínicas de las operadas en libros especiales del servicio. La cátedra de Ginecología fue creada oficialmente por los años de 1911 ó 1912, y se nombró al Dr. Ferráez para desempeñarla. En dicho servicio, fueron cirujanos también los Dres. Siegfried Figueroa Meinhart, Mariano Correa Espinosa, Adolfo González, Felipe Machado Ontiveros, Alberto Rendón Peón, Juan Helguera F., Manuel Espinosa S., y Ramón Osorio C., desempeñando la cátedra de Ginecología desde 1925, el Dr. Rendón Peón, de la Facultad de París. A partir de 1906 han sido cirujanos del Departamento de Hombres los Dres. Saturnino Guzmán Cervera (1891 a 1931), Eudaldo Ferráez, Eduardo Aldana, Alberto Berrón, Hircano Ayuso, Mauro Buenfil, Efraín Gutiérrez Rivas, José Castellanos G., Ernesto Guzmán M., Andrés Peniche C., y Pedro Cámara Milán, éste último desde 1931 hasta la presente fecha, como jefe del servicio y profesor de la cátedra, y los Dres. Carlos López Alonso, Carlos Rubio, Pastor Molina $C$., y Antonio Cabrera, especializados en órganos 


\section{A Cervera-Andrade.}

de los sentidos. Durante algunos años (1925 a 1939) existió en el Hospital un departamento de Odontología, anexo a la Escuela del mismo ramo, y estuvo a cargo del cirujano dentista Dr. Nicolás A Patrón. Los servicio de Otorrinolaringología y radiología, fueron creados casi simultáneamente, durante el año 1931, habiendo estado éste último a cargo, primero, del Dr. Alonso Patrón Gamboa y después el Dr. Cirilo Montes de Oca, hasta hoy. El servicio de Maternidad, que primero ocupó un edificio en el suburbio de la Mejorada, al inaugurarse el nuevo Hospital en 1906, quedó incluido entre los servicios de dicho establecimiento, destinándosele un pabellón del Departamento de Mujeres, habiendo actuado y dejado grata memoria los Dres. Tappam, Arias, Francisco Losa, Carlos Casares (desaparecidos ya del escenario de la vida) y los Dres. Luis Urzaiz, Fernando García y Eduardo Urzaiz Rodríguez Jefe de dicho servicio y profesor de la cátedra respectiva desde el año 1925.

\section{LOS LOGROS EN LA PRIMERA MITAD DEL SIGLO XX.}

Para terminar, haremos la relación cronológica de las operaciones que se hicieron por primera vez, a partir del primer año de este siglo:

Dr. Federico Sauri Reyes: gastroenterostomía por vía transperitoneal, a consecuencia de estenosis pilórica.

Dr. Eduardo Aldana: operó un abocamiento anormal del recto (en la vagina) con imperforación del ano, en una niña pequeña, con éxito.

Dr. Aldana: Citoscopía y cateterismo ureteral (en una señora).

Dr. Eduardo UrLaiz: Punción lumbar.

Dr. Eduardo Urzaiz: Tratamiento de las infecciones de la matriz post-abortum mediante el escobillaje con gasa seca seguido de un toque intrauterino con tintura de yodo -1910 .

Dr. Ernesto Guzmán Méndez: Injerto glandular por el método de Voronof, 1924.

Dr. Alberto Rendón: Operación de la Fort, 1925.

\section{Revista Biomédica}

Dr. Rendón: Histerectomía por vía perineal, 1928.

Dr. Rendón: Nefrectomía por vía transperitoneal.

Dr. Rendón: extirpación de la glándula tiroides, 1929.

Dr. Juan Helguera Fernández: Ventrofijación del muñón resultante de histerectomía subtotal, como tratamiento del prolapso, 1937.

Dr. Mauro Buenfil: Operación de Calot, en una articulación de la cadera anquilosada, 1917.

Dr. Buenfil: Operación de Steinach.-1939.

Esta es, a grandes rasgos, la historia de la cirugía en Yucatán que tengo el honor de someter a la consideración de esta docta asamblea, y si en ella hay algún dato incorrecto, pido se me disculpe, pues, materialmente hasta hoy, ha habido algo de negligencia para ir anotando estos acontecimientos, grandes o pequeños que van jaloneando el progreso de la ciencia y los datos que he recogido, hasta donde fue posible, he procurado ratificarlos unas veces y rectificarlos otras; a pesar de ello pudiera suceder que el día de mañana algún historiador más cuidadoso diga la última palabra. 